Document downloaded from:

http://hdl.handle.net/10251/66009

This paper must be cited as:

ljaz, M.; Ghassemlooy, Z.; Perez, J.; Brazda, V.; Fiser, O. (2013). Enhancing the atmospheric visibility and fog attenuation using a controlled FSO channel. IEEE Photonics Technology Letters. 25(13):1262-1265. doi:10.1109/LPT.2013.2264046

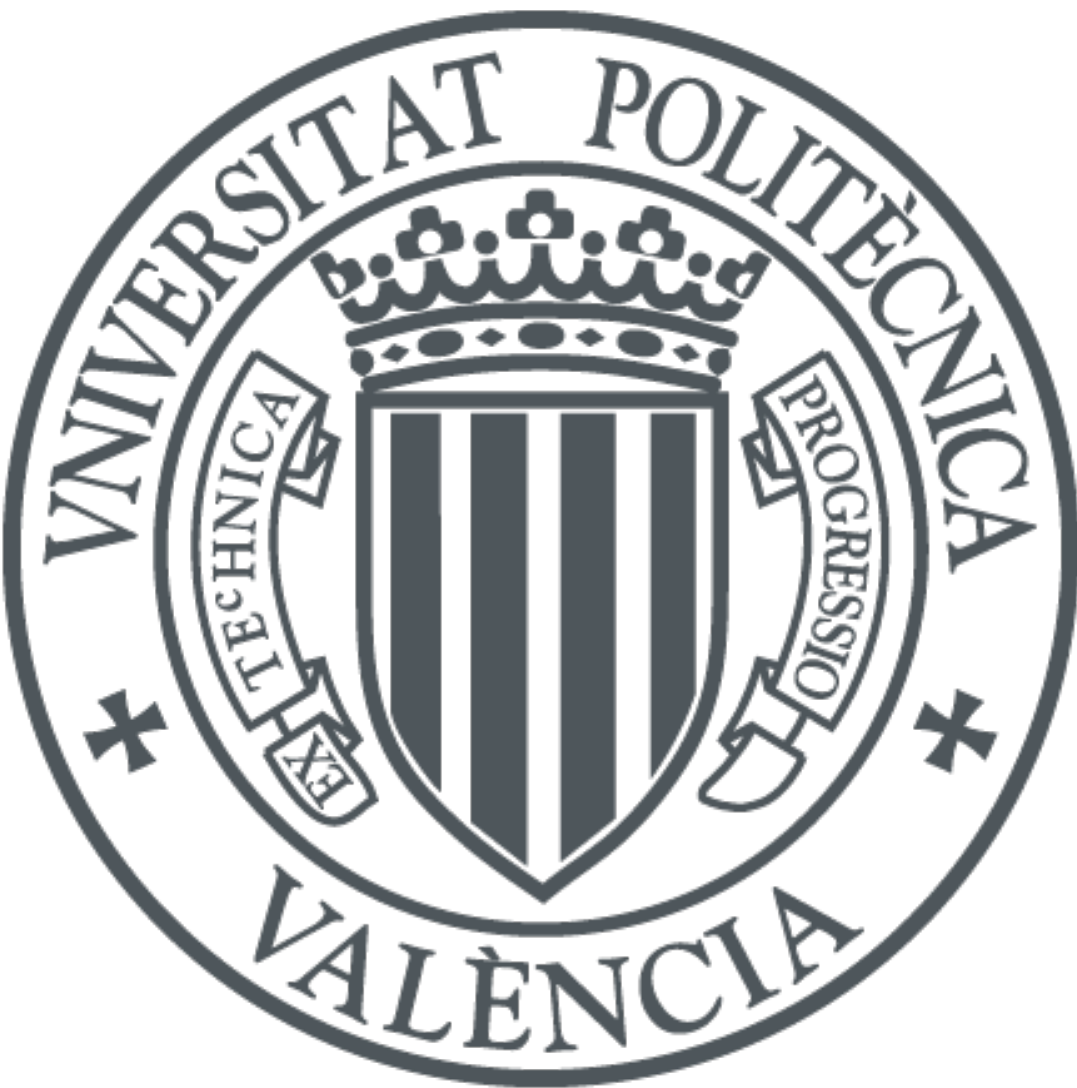

The final publication is available at

http://dx.doi.org/10.1109/LPT.2013.2264046

Copyright Institute of Electrical and Electronics Engineers (IEEE)

Additional Information

(C) 2013 IEEE. Personal use of this material is permitted. Permissíon from IEEE must be obtained for all other uses, in any current or future media, including reprinting/republishing this material for advertisíng or promotional purposes, creating new collective works, for resale or redistribution to servers or lists, or reuse of any copyrighted component of this work in other works. 


\title{
Enhancing the Atmospheric Visibility and Fog Attenuation using a Controlled FSO Channel
}

\author{
Muhammad Ijaz, Student Member, IEEE, Zabih Ghassemlooy, Senior Member, IEEE, \\ Joaquin Perez, Member, IEEE, Vladimir Brazda, and Ondrej Fiser
}

\begin{abstract}
In this paper, two methods for characterizing fog attenuation in terms of atmospheric visibility $V$ in free space optics (FSO) communications are analyzed and compared. Both methods measure the fog attenuation based on $V$ using a charge-coupled device (CCD) camera and a laser-diode at $0.55 \mu \mathrm{m}$. The methods are evaluated in a laboratory controlled FSO atmospheric chamber operating at individual wavelengths of $0.83,1.31$ and $1.55 \mu \mathrm{m}$ and using a continuous optical spectrum range of 0.6 to $1.6 \mu \mathrm{m}$. The $C C D$ technique shows great accuracy for $V<50 \mathrm{~m}$ and the laser technique for the range beyond $100 \mathrm{~m}$, thus allowing enhancement of the characterization of FSO links in thick and dense fog conditions. The experimental results also indicate a wavelength dependency for $V$ below $0.5 \mathrm{~km}$ where the attenuation decreases from $52 \mathrm{~dB} / \mathrm{km}$ at visible wavelengths to $46 \mathrm{~dB} / \mathrm{km}$ at near infrared (NIR) wavelengths.
\end{abstract}

Index Terms - Free space optics; Fog attenuation; Visibility

\section{INTRODUCTION}

$I^{1}$ $\mathrm{n}$ recent years, the demand for FSO communications has increased considerably due to offering large capacity usage (data, voice, and video) in a number of short to medium link range applications [1]. FSO offers a wide modulation bandwidth and an aggregate data rate of over $100 \mathrm{~Gb} / \mathrm{s}$ using wavelength division multiplexing (WDM) [2]. It consumes a low power, provides much improved security against electromagnetic interference (EMI) and does not require administrative licensing or tariffs [3]. Therefore, FSO is considered as an alternative option in metropolitan and local area networks ((M/L)ANs) as well as a viable solution to the access network "last mile" problem in which the compromise between available data rates and the cost is desirable [4]. However, the performance of the FSO system is dependent on atmospheric conditions particularly in the presence of aerosols, e.g., fog, smoke, and dust, which results in scattering and attenuation [5]. For example, in a dense fog condition

Manuscript submitted October 30, 2012. This work was supported by European COST actions ( IC801 and IC1101) and by the grant of the Agency of the Czech Republic GACR P102/11/1376.

M. Ijaz, Z. Ghassemlooy and J. Perez are with the Optical Communications Research Group, Faculty of Engineering and Environment, Northumbria University, NE1 8ST, Newcastle Upon Tyne, UK (e-mail: \{muhammad.ijaz, z.ghassemlooy\}@northumbria.ac.uk).

V. Brazda is with the Faculty of Electronics and Informatics, University of Pardubice, Studentska 95, 53210 Pardubice, Czech Republic.

O. Fiser is with the Department of Meteorology at Institute of Atmospheric Physics AS CR, Bocni II, 1401,141 31 Prague 4, Czech Republic.

Copyright (c) 2012 IEEE. Personal use of this material is permitted. However, permission to use this material for any other purposes must be obtained from the IEEE by sending a request to pubs-permissions@ieee.org. (defined by $V<0.5 \mathrm{~km}$ ) the FSO link failure is high due to the scattering and absorption of propagating optical beam, which is not desirable by the end users [6]. As a result the study of the relationship between the atmospheric aerosols and FSO wavelengths for thick and dense fog conditions are important in order to enhance the FSO link performance and therefore improve the link availability [7]. However, due to the difficulty of measuring the particle density and the shape of aerosols in the atmosphere, measurement of $V$ is the most common technique used to characterize the fog attenuation in FSO links. Currently, the most accepted method to measure $V$ is based on the proportional amount of scattered light captured by the optical receiver. Determining $V$ from CCD images based on the edge detection of various distant objects in the image is an alternative technique that has been employed to complement the visibility measurement at airports [8].

In this paper both techniques are compared and experimentally evaluated in a dedicated indoor atmospheric chamber that enables us to characterize the atmospheric fog and the FSO link performance, likewise to turbulence as outlined in [9]. The main objective of this paper is to provide a complete analysis and verification of the enhanced visibility measurements for FSO under homogeneous fog conditions. Therefore, precise measurements of the concurrent visibility and attenuation $(\mathrm{dB} / \mathrm{km})$ along the length of the FSO link can be carried out for each wavelengths of $0.83,1.31$ and $1.55 \mu \mathrm{m}$ for dense fog $(V<0.5 \mathrm{~km})$ to light fog conditions $(V>0.5 \mathrm{~km})$. Moreover, we have carried out real time measurements, taken in identical conditions, for the complete visible - NIR spectrum in order to verify the wavelength dependency of the fog attenuation for $V<0.5 \mathrm{~km}$.

This paper is organised as follows: the characterization of the fog attenuation in laboratory chamber is outlined in Section II, whereas the experimental description is explained in Section III. In Section IV experimental results and discussions are presented. The conclusions are drawn in Section $V$.

\section{Characterization OF Fog ATtENUATION IN LABORATORY CHAMBER}

$V$ is the visual range where a contrast ratio $\left(C / C_{o}\right)$ drops to the visual threshold $T_{t h}$ of $2 \%$ along the propagation path, where $C_{o}$ is the intrinsic contrast of the object and $C$ is the contrast measured at the propagation distance $L(\mathrm{~km})[10]$. Using Koschmieder relation [11] and Beer-Lambert-Boguer's law, $V$ is given by : 


$$
V=\frac{\ln (0.02)}{\ln \left(\frac{C}{C_{o}}\right)} L .
$$

In this case $V$ can also be expressed in terms of the atmospheric attenuation coefficient $\beta_{\lambda}$ and $T_{\text {th }}$ at a wavelength of $0.55 \mu \mathrm{m}$ and is given as $[12,13]$ :

$$
V=-\frac{10 \log _{10}\left(T_{t h}\right)}{\beta_{\lambda}},
$$

where $\beta_{\lambda}(\mathrm{dB} / \mathrm{km})$ is the total attenuation due to the absorption and scattering of light, which is theoretically investigated in [14]. Generally, ROF induced optical attenuation at a given wavelength $\lambda$ can be predicted using empirical models. Kim model, relating $V$ with the ROF attenuation for the visible NIR wavelengths and is defined as [15]:

$$
V=\frac{10 \log _{10} T_{t h}}{\beta_{\lambda}}\left(\frac{\lambda}{\lambda_{0}}\right)^{-q}
$$

where $\lambda_{0}=0.55 \mu \mathrm{m}$ is the maximum sensitive wavelength for the human eye. $q$ is proportional to the particle size distribution and is related to $V$ [16]. Kim used $q=0$ to indicate that the atmospheric attenuation coefficient $\beta_{\lambda}$ is wavelength independent for dense fog conditions $(V<0.5 \mathrm{~km})$, can be found in [15].

\section{EXPERIMENT DESCRIPTION}

The block diagram of proposed laboratory based FSO link is shown in Fig. 1 (a). An optical transmitter $T_{x}$ and an optical receiver $R_{x}$ are separated by the channel, which is represented by the atmospheric chamber of a dimension of $55 \mathrm{~cm} \times 30 \mathrm{~cm} \times 30 \mathrm{~cm}$, see Fig. 1(b). Fog is generated using a commercial fog machine (water steam) with $100 \%$ humidity to mimic ROF [17]. The amount of fog in the atmospheric chamber is allowed to settle down homogeneously and is controlled by fans and ventilation systems as illustrated in Fig. 1(c).

Following (1), the experiment was performed using a CCD camera (model GE X5, 14 megapixels) in the presence of two optical links at $0.83 \mu \mathrm{m}$ and $1.55 \mu \mathrm{m}$. We placed a black and white target at the receiver. The ratio $C / C_{o}$ between the black and white areas was computed by measuring the luminance of the white $L_{w}$ and black $L_{b}$ parts of the target, where $C_{o}$ is the contrast without fog and $C$ is the contrast with fog $[10,12]$ :

$$
C=\frac{L_{w}-L_{b}}{L_{w}+L_{b}}
$$

Following (2), the experiment was performed using the laser diode at $0.55 \mu \mathrm{m}$ in presence of optical links at $0.83,1.31$ and $1.55 \mu \mathrm{m}$. The normalized transmittance $T$ was calculated at $R_{x}$ before and after the injection of fog into the chamber for all wavelengths. We measured $\beta_{\lambda}$ corresponding to the measured $T$ from light to dense fog conditions. This allows us to measure the link $V$ at $0.55 \mu \mathrm{m}$ simultaneously with the fog attenuation at $0.83,1.31$ and $1.55 \mu \mathrm{m}$ along the length of the chamber.

The same experiment was performed using a continuous tungsten halogen light source, model LS-1 from Ocean Optics Inc., with a broad spectrum (0.36 to $2.5 \mu \mathrm{m})$ and an optical

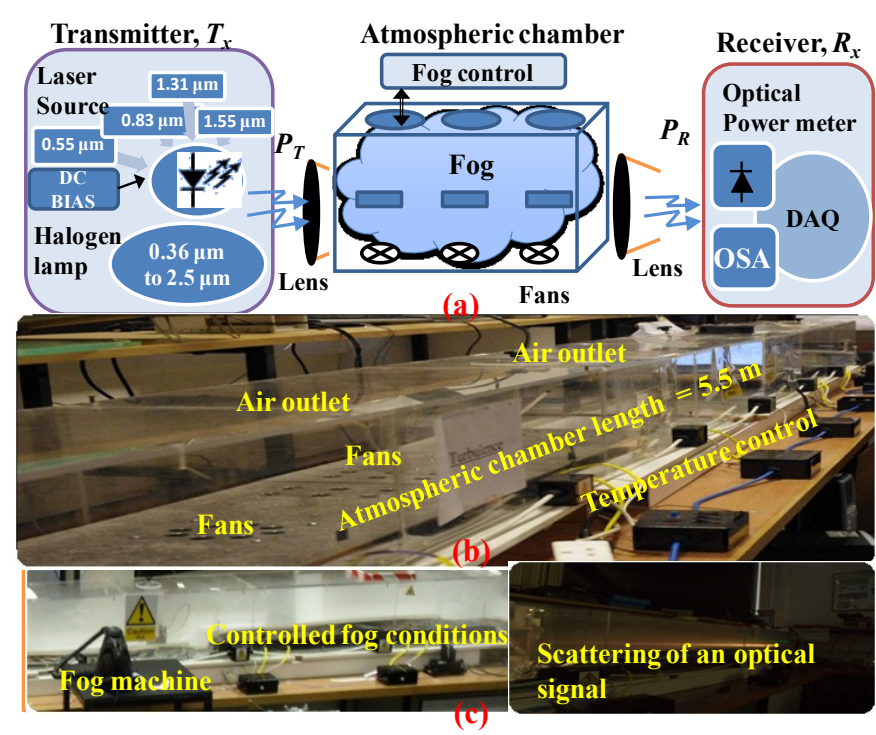

Figure 1. (a) The block diagram of the experimental setup, (b) the laboratory controlled atmospheric channel, and (c) the scattering of an optical signal due to the fog in the chamber.

receiver $R_{x}$ using an Anritsu MS9001B1 optical spectrum analyzer (OSA) with a spectral response of 0.6 to $1.75 \mu \mathrm{m}$. This allows us to measure the fog attenuation for different values of $V$ across the spectrum of interest simultaneously. The data is acquired by an automated data acquisition (DAQ) system driven by the LabVIEW environment. The geometric and other losses were not taken into account for $T_{x}$, as the normalized $P_{R}$ was measured both before and after the fog at $R_{x}$ to attain the wavelength dependent fog loss.

\section{EXPERIMENTAL RESULTS AND DISCUSSIONS}

\section{A. Characterization of Fog Attenuation}

Fog is a random dynamic atmospheric phenomenon and the probability of occurrence of the dense fog in the real outdoor atmosphere is very low. Fig. 2 illustrate the typical measured distribution of $V$ from two field experiments at the Newcastle airport, UK and Milesovka Hill, Czech Republic over a year and is compared to the average data generated from two experiments over one hour time period using the laboratory atmospheric chamber. The minimum $V$ achieved from the data at Milesovka and Newcastle airport are $33 \mathrm{~m}$ for $0.1 \%$ of the year and $50 \mathrm{~m}$ for $0.01 \%$ of the year, respectively. However, using the atmospheric chamber allows us to achieve a very dense fog condition with $V=33 \mathrm{~m}$ for $1 \%$ of the time, which demonstrates our system advantage to replicate outdoor dense fog field trial conditions without waiting for a year.

Fig. 3(a) shows the plots for the concurrent measured $V$ and the fog attenuation (in $\mathrm{dB} / \mathrm{km}$ ) at $0.83 \mu \mathrm{m}$ and $1.55 \mu \mathrm{m}$ using $\mathrm{CCD}$ and a laser diode at $0.55 \mu \mathrm{m}$. Moreover, Kim model is compared with the experimental data in order to validate the accuracy of the experiment in the atmospheric chamber. Fig 3(b) depicts the picture captured by CCD to measure $V$ in light (left) and dense (right) fog conditions. CCD technique shows higher accuracy for $V<0.05 \mathrm{~km}$ and the laser technique for the range $>0.1 \mathrm{~km}$, see Fig. 3(a). Therefore, these techniques allow enhancement of the characterization of 


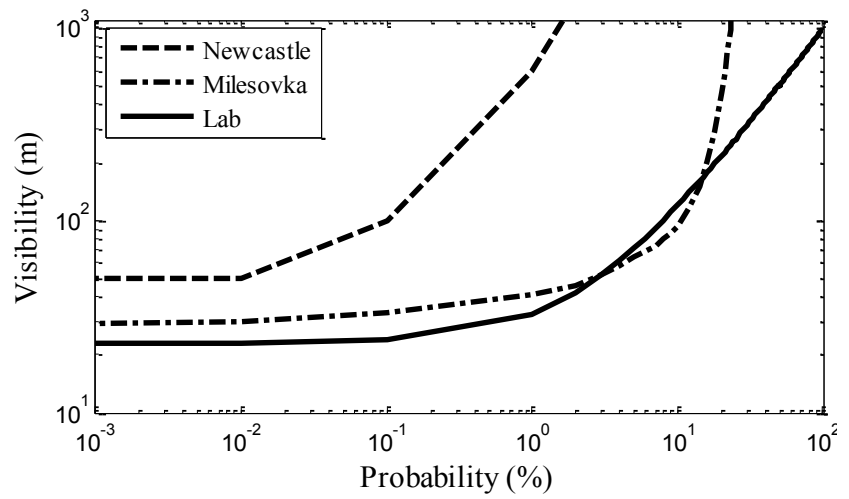

Figure 2. Comparison of the measured visibility for the outdoor and atmospheric chamber.
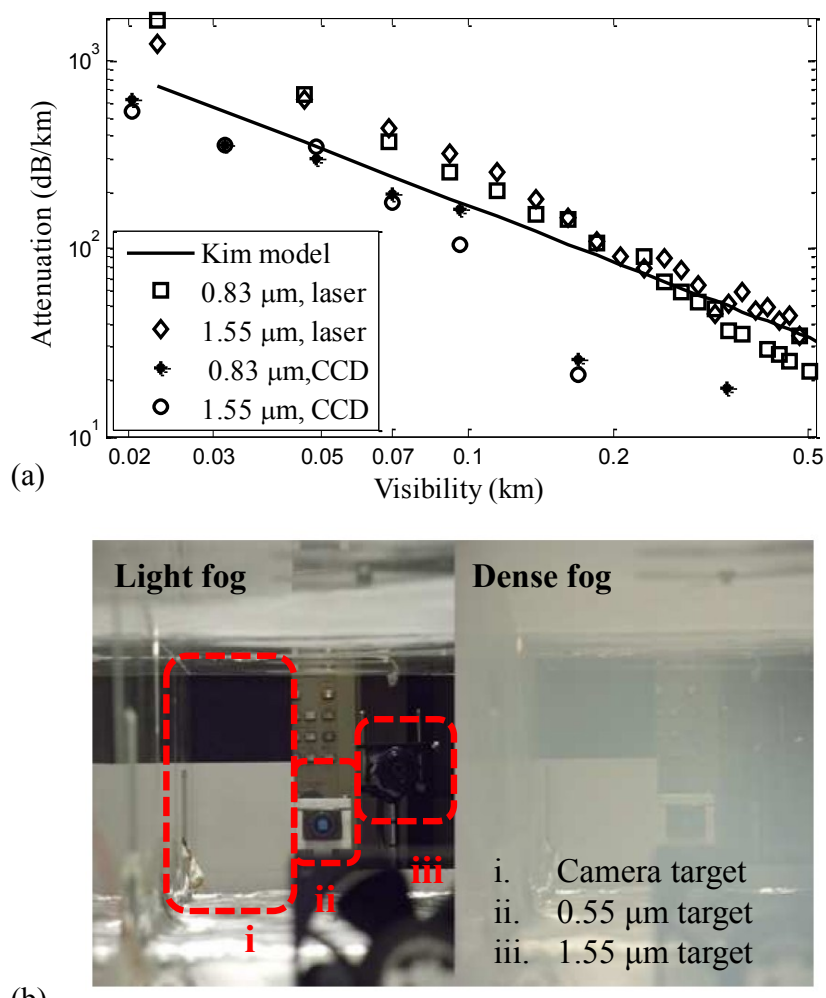

(b)

Figure 3. (a) The characterization of measured visibility and fog attenuation using CCD, laser diodes and Kim model, and (b) CCD fog measurements snapshots with light (left) and dense (right) fog conditions, both at the atmospheric chamber.

an FSO link in thick and dense fog conditions. The measured $V$, using both methods, is in close agreement with Kim model. This validates the accuracy of visibility measurement and the corresponding fog attenuation in the chamber.

The dependence of the wavelength on the fog attenuation is verified for $V<0.5 \mathrm{~km}$ by using selective wavelengths. Plots of measured fog attenuation against the measured $V$ for $0.83 \mu \mathrm{m}, 1.31 \mu \mathrm{m}$ and $1.55 \mu \mathrm{m}$ wavelengths are shown in Fig. 4. The measured attenuation is wavelength dependent at $0.83 \mu \mathrm{m}, 1.31 \mu \mathrm{m}$ and $1.55 \mu \mathrm{m}$ for the dense fog condition, i.e., $V<0.5 \mathrm{~km}$. The measured attenuation at $1.31 \mu \mathrm{m}$ is higher than at $1.55 \mu \mathrm{m}$ for $V<0.5 \mathrm{~km}$, contradicting the wavelength independency of Kim model. The experimental results show the inconsistent effect of fog attenuation on the selected wavelengths for $V<0.5 \mathrm{~km}$.

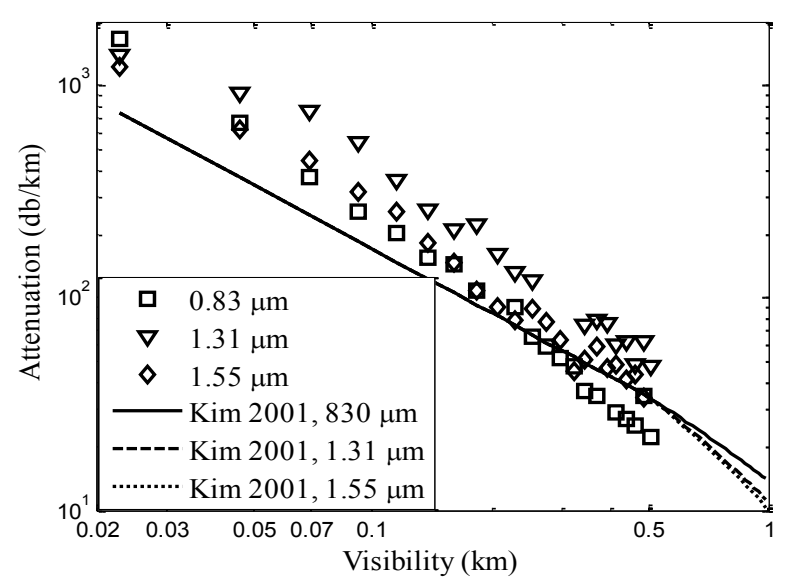

Figure 4. Comparison of measured fog attenuation for different wavelengths.

\section{B. Comparison of Attenuation Spectrum for Fog}

Fig. 5 illustrates the fog attenuation for the visible - NIR spectrum at dense fog conditions $(V=0.357 \mathrm{~km})$ in the atmospheric chamber. The measured fog attenuation for the visible range is $52 \mathrm{~dB} / \mathrm{km}$, decreasing to $46 \mathrm{~dB} / \mathrm{km}$ for the NIR range of the spectrum. However, the peak attenuation in the wavelength range of $1.35-1.45 \mu \mathrm{m}$ is due to strong water absorption, which is consistent with the published results as in [18]. The measured fog attenuation for the visible - NIR spectrum is also compared to the wavelength independent Kim model for $V<0.5 \mathrm{~km}$. The experimental results contradict the wavelength independent fog attenuation for the spectral attenuation.

The measured fog attenuation for the light fog $(V \sim 0.783 \mathrm{~km})$ for the visible - NIR spectrum is depicted in

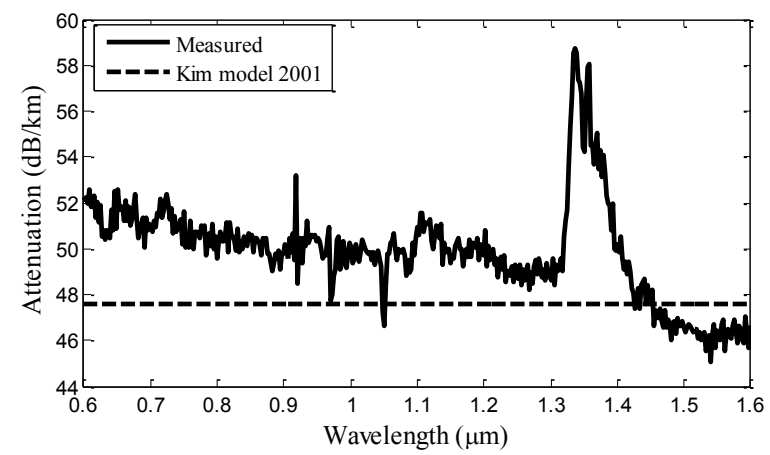

Figure 5. Comparison of measured fog attenuation $(\mathrm{dB} / \mathrm{km})$ with Kim model for the visible - NIR spectrum at $V=0.357 \mathrm{~km}$.

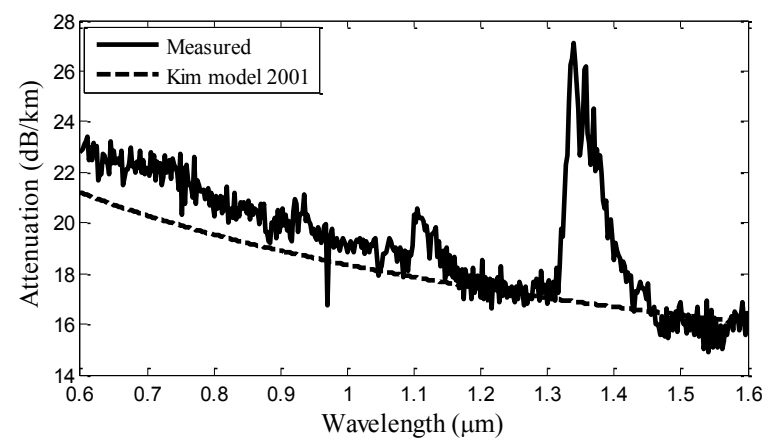

Figure 6. Comparison of measured fog attenuation $(\mathrm{dB} / \mathrm{km})$ with Kim model for the visible - NIR spectrum at $V=0.785 \mathrm{~km}$. 
Fig. 6. The measured attenuation spectrum is very close to Kim model at $V=0.783 \mathrm{~km}$. This validates that Kim model is more realistic to use when $V>0.5 \mathrm{~km}$. This model does not take into account the wavelength for $V<0.5 \mathrm{~km}$. However, the experimental data shows that the spectral attenuation is wavelength dependent for $V<0.5 \mathrm{~km}$. This validates that the Kim model need to be revised for $V<0.5 \mathrm{~km}$ to predict the wavelength dependent fog attenuation.

\section{CONCLUSION}

In this paper, we have implemented and compared different methods using CCD and laser technique to measure $V$ and characterize the fog attenuation measurement under laboratory dense fog conditions. The laboratory indoor atmospheric chamber is calibrated to ROF conditions by using Kim model. The visibility measurement using CCDs and the laser technique are very accurate for the link spans of $<0.05 \mathrm{~km}$ and $<0.1 \mathrm{~km}$, respectively. This accuracy is essential when implementing and installing real-time low cost visibility system where accuracy and early response are crucial, e.g. road surveillance, airport security, local weather applications. Moreover, we have compared individual wavelengths of $0.83 \mu \mathrm{m}, 1.31 \mu \mathrm{m}$ and $1.55 \mu \mathrm{m}$ in the homogeneous fog conditions. We have verified that the fog attenuation is wavelength dependent for all the spectrum range of $0.6 \mu \mathrm{m}<\lambda<1.6 \mu \mathrm{m}$ under a controlled fog condition for $V<0.5 \mathrm{~km}$.

\section{REFERENCES}

[1] E. Ciaramella, Y. Arimoto, G. Contestabile, M. Presi, A. D'Errico, V. Guarino, and M. Matsumoto, "1.28 terabit/s (32x40 Gbit/s) wdm transmission system for free space optical communications," Selected Areas in Communications, IEEE Journal on, vol. 27, pp. 1639-1645, 2009.

[2] N. Cvijetic, Q. Dayou, Y. Jianjun, H. Yue-Kai, and W. Ting, "100 Gb/s per-channel free-space optical transmission with coherent detection and MIMO processing," in 35th European Conference on Optical Communication ECOC, pp. 1-2, 2009.

[3] Z. Ghassemlooy, W. Popoola, and S. Rajbhandari, Optical wireless communications : system and channel modelling with MATLAB. Boca Raton, FL: CRC Press, 2012
[4] E. Leitgeb, M. Gebhart, and U. Birnbacher, "Optical networks, last mile access and applications," Journal of Optical and Fiber Communications Research, vol. 2, pp. 56-85, 2005.

[5] J. C. Ricklin, S. M. Hammel, F. D. Eaton, and S. L. Lachinova, "Atmospheric channel effects on free-space laser communication," Journal of Optical and Fiber Communications Research, vol. 3, pp. 111158, 2006.

[6] J. Perez, Z. Ghassemlooy, S. Rajbhandari, M. Ijaz, and H. L. Minh, "Ethernet FSO Communications Link Performance Study Under a Controlled Fog Environment," IEEE Communications Letters, vol. 16, pp. 408-410, 2012.

[7] M. Grabner and V. Kvicera, "The wavelength dependent model of extinction in fog and haze for free space optical communication," Opt. Express, vol. 19, pp. 3379-3386, 2011.

[8] D. Bäumer, S. Versick, and B. Vogel, "Determination of the visibility using a digital panorama camera," Atmospheric Environment, vol. 42, pp. 2593-2602, 2008.

[9] Z. Ghassemlooy, H. Le Minh, S. Rajbhandari, J. Perez, and M. Ijaz, "Performance Analysis of Ethernet/Fast-Ethernet Free Space Optical Communications in a Controlled Weak Turbulence Condition," Journal of Lightwave Technology, vol. 30, pp. 2188- 2194, 2012.

[10] C. P. Colvero, M. C. R. Cordeiro, and J. P. von der Weid, "Real-time measurements of visibility and transmission in far mid and near-IR free space optical links," Electronics Letters, vol. 41, pp. 610-611, 2005.

[11] H. Koschmieder, "Theorie der horizontalen Sichtweite," Beitrage zur physik der freien Atmosphare, vol. 12, pp. 33-53, 1924.

[12] H. Weichel, "Laser beam propagation in the atmosphere.," Bellingham: SPIE Optical Engineering, vol. TT3, pp. 25-39, 1990.

[13] A. K. Majumdar and J. C. Ricklin, "Free-Space Laser Communications: Principles and Advances," Springer Science, vol. LLC, 233, New York, USA, 2008.

[14] M. Ijaz, Z. Ghassemlooy, S. Rajbhandari, H. Le Minh, J. Perez, and A. Gholami, "Comparison of $830 \mathrm{~nm}$ and $1550 \mathrm{~nm}$ based free space optical communications link under controlled fog conditions," in 8th International Symposium on Communication Systems, Networks \& Digital Signal Processing (CSNDSP), pp. 1-5, 2012.

[15] I. I. Kim, B. McArthur, and E. Korevaar, "Comparison of laser beam propagation at $785 \mathrm{~nm}$ and $1550 \mathrm{~nm}$ in fog and haze for optical wireless communications," in Proc. SPIE 4214, Boston, MA, USA, 2001.

[16] P. W. Kruse, L. D. McGlauchlin, and E. B. McQuistan, Elements of infrared technology: Generation, transmission and detection. New York: John Wiley and Sons, 1962.

[17] M. S. Awan, L. C. Horwath, S. S. Muhammad, E. Leitgeb, F. Nadeem, and M. S. Khan, "Characterization of fog and snow attenuations for freespace optical propagation," Journal of Communications, vol. 4, pp. 533545, 2009.

[18] R. N. Clark, "Spectroscopy of rocks and minerals, and principles of spectroscopy," in Manual of remote sensing. vol. 3, ed New York: John Wiley \& Sons, Inc., pp. 3-58, 1999. 